\title{
Constraints on scalar diffusion anomaly in three-dimensional flows having bounded velocity gradients
}

\author{
Chuong V. Tran ${ }^{1}$ \\ School of Mathematics and Statistics, University of St Andrews \\ St Andrews KY16 9SS, United Kingdom
}

\begin{abstract}
This study is concerned with the decay behaviour of a passive scalar $\theta$ in three-dimensional flows having bounded velocity gradients. Given an initially smooth scalar distribution, the decay rate $d\left\langle\theta^{2}\right\rangle / d t$ of the scalar variance $\left\langle\theta^{2}\right\rangle$ is found to be bounded in terms of controlled physical parameters. Furthermore, in the zero diffusivity limit, $\kappa \rightarrow 0$, this rate vanishes as $\kappa^{\alpha_{0}}$ if there exists an $\alpha_{0} \in(0,1]$ independent of $\kappa$ such that $\left\langle\left|(-\Delta)^{\alpha / 2} \theta\right|^{2}\right\rangle<\infty$ for $\alpha \leq \alpha_{0}$. This condition is satisfied if in the limit $\kappa \rightarrow 0$, the variance spectrum $\Theta(k)$ remains steeper than $k^{-1}$ for large wave numbers $k$. When no such positive $\alpha_{0}$ exists, the scalar field may be said to become virtually singular. A plausible scenario consistent with Batchelor's theory is that $\Theta(k)$ becomes increasingly shallower for smaller $\kappa$, approaching the Batchelor scaling $k^{-1}$ in the limit $\kappa \rightarrow 0$. For this classical case, the decay rate also vanishes, albeit more slowly — like $\left(\ln P_{r}\right)^{-1}$, where $P_{r}$ is the Prandtl or Schmidt number. Hence, diffusion anomaly is ruled out for a broad range of scalar distribution, including power-law spectra no shallower than $k^{-1}$. The implication is that in order to have a $\kappa$-independent and non-vanishing decay rate, the variance at small scales must necessarily be greater than that allowed by the Batchelor spectrum. These results are discussed in the light of existing literature on the asymptotic exponential decay $\left\langle\theta^{2}\right\rangle \sim e^{-\gamma t}$, where $\gamma>0$ is independent of $\kappa$.
\end{abstract}

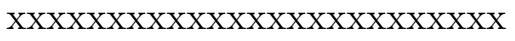

\section{Introduction}

The transport and diffusion of a passive scalar $\theta(\boldsymbol{x}, t)$ in incompressible fluid flows $\boldsymbol{u}(\boldsymbol{x}, t)$ is governed by the advection-diffusion equation,

$$
\partial_{t} \theta+\boldsymbol{u} \cdot \nabla \theta=\kappa \Delta \theta, \quad \nabla \cdot \boldsymbol{u}=0
$$

where $\kappa$ is the diffusivity. The incompressible flows $\boldsymbol{u}$ may be described by a separate system of equations, for example the Navier-Stokes system, or just satisfy some prescribed conditions. This study considers three-dimensional flows having bounded velocity gradients, i.e. $|\nabla \boldsymbol{u}|<\infty$. For simplicity, periodic boundary conditions are imposed on both $\boldsymbol{u}$ and $\theta$, and the scalar spatial average is set to zero, i.e. $\langle\theta\rangle=0$. The initial scalar distribution is assumed to be smooth. The diffusive decay of the scalar variance $\left\langle\theta^{2}\right\rangle$ is governed by

$$
\frac{d}{d t}\left\langle\theta^{2}\right\rangle=-2 \kappa\left\langle|\nabla \theta|^{2}\right\rangle
$$

A problem of long-standing interest is to what extent this decay can be accelerated as the scalar field becomes "less smooth" through advective amplification of its gradients.

\footnotetext{
${ }^{1}$ chuong@mcs.st-and.ac.uk
} 
This problem, together with related issues concerning the scalar spectrum, has been actively studied for decades, ${ }^{1-21}$ most notably since the seminal work of Batchelor and coworkers. ${ }^{3,4}$ In one of these studies, Batchelor ${ }^{3}$ considered presumably non-singular Navier-Stokes flows, where the fluid viscosity $\nu$ is much greater than the scalar diffusivity $\kappa$. This so-called regime of large Prandtl or Schmidt number $P_{r}$, defined by $P_{r}=\nu / \kappa$, is of both practical and theoretical interest, where a fundamental question is whether the decay rate $d\left\langle\theta^{2}\right\rangle / d t$ would remain non-zero in the limit $\kappa \rightarrow 0$. The general belief, having its root in the phenomenological theory of turbulence, is that this rate becomes independent of $\kappa$ and remains non-zero in that limit (even for two-dimensional flows, which are presumably poorer stirrers as compared with their three-dimensional counterparts). Thus, the scalar variance is believed to cascade to the small scales and undergo anomalous diffusion just as the fluid kinetic energy. However, doubts have been cast upon this picture. ${ }^{11,16}$ In particular, Sreenivasan ${ }^{16}$ suggested that the analogy between the energy and scalar variance is rather weak and questioned whether there is a passive scalar cascade at all (for a discussion see Chapter 5 of Davidson's book). ${ }^{22}$ Recently, Tran and Dritschel ${ }^{23}$ showed that for general two-dimensional flows having finite enstrophy and power-law spectra of $\left\langle\theta^{2}\right\rangle$ no shallower than $k^{-1}$, the decay rate $2 \kappa\left\langle|\nabla \theta|^{2}\right\rangle$ vanishes in the limit $\kappa \rightarrow 0$. Their calculation took full account of the suppression of scalar gradients by diffusive effects.

This study extends the above result to the three-dimensional case, with an improvement in the method of analysis by making use of an analytic inequality. For flows having bounded velocity gradients, the maximally achievable decay rate $2 \kappa\left\langle|\nabla \theta|^{2}\right\rangle$ is shown to be bounded from above. Furthermore, in the limit $\kappa \rightarrow 0$, the derived upper bound vanishes provided that the scalar field remains marginally smooth. More precisely, if there exists a positive $\alpha_{0}$ independent of $\kappa$ such that $\left\langle\left|(-\Delta)^{\alpha / 2} \theta\right|^{2}\right\rangle<\infty$ for $\alpha \leq \alpha_{0}$, then $\kappa\left\langle|\nabla \theta|^{2}\right\rangle \leq C \kappa^{\alpha_{0}}$. Here, $\alpha_{0} \in(0,1]$ and $C$ is a constant, depending on $|\nabla \boldsymbol{u}|$ and $\left\langle\left|(-\Delta)^{\alpha_{0} / 2} \theta\right|^{2}\right\rangle$, both being bounded and independent of $\kappa$. This constraint covers all scalar distribution having variance spectrum $\Theta(k)$ steeper than $k^{-1}$ at the spectral tail, i.e. at large wave numbers $k$. This is an improvement over the result of Tran and Dritschel ${ }^{23}$ for the two-dimensional case, where power-law spectra were assumed explicitly in their calculations. When no such positive $\alpha_{0}$ exists, a plausible scenario consistent with Batchelor's theory ${ }^{3}$ is that $\Theta(k)$ becomes increasingly shallower for smaller $\kappa$, approaching the Batchelor scaling $k^{-1}$ in the limit $\kappa \rightarrow 0$. For this classical case, the semi-analytic estimate of Tran and Dritschel can be applied, yielding a vanishing decay rate no slower than $\left(\ln P_{r}\right)^{-1}$. Hence, diffusion anomaly is ruled out for a broad class of scalar distribution, including power-law spectra no shallower than $k^{-1}$. The implication is that a non-vanishing decay rate in the limit $\kappa \rightarrow 0$ necessarily requires the variance at small scales to be greater than that allowed by the Batchelor spectrum. This is consistent with recent studies ${ }^{1,7,14,19}$ suggesting spectra shallower than $k^{-1}$. This analysis when applied to the forced case implies that for power-law $\Theta(k)$, the scalar variance diverges in the limit $\kappa \rightarrow 0$. Finally, the present results are discussed in the light of existing literature $8,13,17,20$ on the exponential decay behavior at large times, when the exponential decay rate becomes independent of $\kappa$ and remains non-zero.

\section{Basic estimates}

This section briefly recalls a basic calculus inequality and related estimates, both being used in this study. In what follows, $\theta$ is bounded and assumed to be sufficiently smooth so that the quantities 
under consideration are well defined.

The Fourier representation of $\theta(\boldsymbol{x}, t)$ is

$$
\theta(\boldsymbol{x})=\sum_{\boldsymbol{k}} \widehat{\theta}(\boldsymbol{k}) \exp \{i \boldsymbol{k} \cdot \boldsymbol{x}\}
$$

where $\widehat{\theta}(\boldsymbol{k})$ is the Fourier transform of $\theta(\boldsymbol{x})$ and $\boldsymbol{k}$ is the wave vector. In (3), the time variable has been suppressed for convenience. For real $\alpha$, including negative values, the fractional derivative $(-\Delta)^{\alpha / 2}$ is defined by

$$
(-\Delta)^{\alpha / 2} \theta(\boldsymbol{x})=\sum_{\boldsymbol{k}} k^{\alpha} \widehat{\theta}(\boldsymbol{k}) \exp \{i \boldsymbol{k} \cdot \boldsymbol{x}\},
$$

where $k=|\boldsymbol{k}|$ is the wave number. For $\alpha \leq 1$, the following interpolation-type inequality holds

$$
\left\langle|\nabla \theta|^{2}\right\rangle \leq\left\langle|\Delta \theta|^{2}\right\rangle^{(1-\alpha) /(2-\alpha)}\left\langle\left|(-\Delta)^{\alpha / 2} \theta\right|^{2}\right\rangle^{1 /(2-\alpha)} .
$$

This inequality reduces to the Cauchy-Schwarz inequality and an identity for $\alpha=0$ and $\alpha=1$, respectively. For $\alpha<1$, Eq. (5) can be proved by an elementary method, ${ }^{24,25}$ and the proof goes as follows. One has

$$
\begin{aligned}
\int k^{2} \Theta(k) d k & =\int\left(k^{4} \Theta(k)\right)^{(1-\alpha) /(2-\alpha)}\left(k^{2 \alpha} \Theta(k)\right)^{1 /(2-\alpha)} d k \\
& \leq\left(\int k^{4} \Theta(k) d k\right)^{(1-\alpha) /(2-\alpha)}\left(\int k^{2 \alpha} \Theta(k) d k\right)^{1 /(2-\alpha)},
\end{aligned}
$$

where the Hölder inequality with the pair of conjugate exponents $p=(2-\alpha) /(1-\alpha)$ and $q=2-\alpha$ has been used. Eq. (6) is equivalent to (5), thus proving (5). For convenient application in the subsequent sections, let us rewrite (5) as $\left\langle|\nabla \theta|^{2}\right\rangle^{(2-\alpha) /(1-\alpha)} \leq\left\langle|\Delta \theta|^{2}\right\rangle\left\langle\left|(-\Delta)^{\alpha / 2} \theta\right|^{2}\right\rangle^{1 /(1-\alpha)}$ and re-arrange the factors to obtain

$$
\frac{\left\langle|\nabla \theta|^{2}\right\rangle^{2}}{\left\langle|\Delta \theta|^{2}\right\rangle} \leq \frac{\left\langle\left|(-\Delta)^{\alpha / 2} \theta\right|^{2}\right\rangle^{1 /(1-\alpha)}}{\left\langle|\nabla \theta|^{2}\right\rangle^{\alpha /(1-\alpha)}} .
$$

Equation (7) implies that if there exists an $\alpha_{0}>0$ such that $\left\langle\left|(-\Delta)^{\alpha_{0} / 2} \theta\right|^{2}\right\rangle<\infty$ for $\alpha \leq \alpha_{0}$, then the ratio on its left-hand side vanishes in the limit $\left\langle|\nabla \theta|^{2}\right\rangle \rightarrow \infty$ because the right-hand side vanishes in this limit. This is the case if $\Theta(k)$ is steeper than $k^{-1}$ for large $k$.

For the critical spectrum $\Theta(k) \propto k^{-1}$ extending to infinity, no such $\alpha_{0}$ exists. Nevertheless, the ratio $\left\langle|\nabla \theta|^{2}\right\rangle^{2} /\left\langle|\Delta \theta|^{2}\right\rangle$ also vanishes for bounded $\left\langle\theta^{2}\right\rangle$. Indeed, if $\Theta(k)=c k^{-1}$ for $k \in\left[k_{1}, k_{2}\right]$ (where $k_{1} \ll k_{2}$ ) and negligible otherwise (steeper than $k^{-1}$ for $k>k_{2}$ ), then $c=\left\langle\theta^{2}\right\rangle / \ln \left(k_{2} / k_{1}\right)$. It follows that

$$
\frac{\left\langle|\nabla \theta|^{2}\right\rangle^{2}}{\left\langle|\Delta \theta|^{2}\right\rangle}=\frac{\left(c \int_{k_{1}}^{k_{2}} k d k\right)^{2}}{c \int_{k_{1}}^{k_{2}} k^{3} d k}=\frac{\left\langle\theta^{2}\right\rangle}{\ln \left(k_{2} / k_{1}\right)} .
$$

The limit $k_{2} \rightarrow \infty$ necessarily entails $\ln \left(k_{2} / k_{1}\right) \rightarrow \infty$ if $k_{1}$ remains bounded. Hence $\left\langle|\nabla \theta|^{2}\right\rangle^{2} /\left\langle|\Delta \theta|^{2}\right\rangle \rightarrow$ 0 as claimed. Note that the inclusion of the contribution from $k>k_{2}$, where $\Theta(k)$ is steeper than $k^{-1}$, does not alter (8) in any significant manner (see Tran and Dritschel ${ }^{23}$ for details).

The above results imply that given a finite field $\theta$, the ratio $\left\langle|\nabla \theta|^{2}\right\rangle^{2} /\left\langle|\Delta \theta|^{2}\right\rangle$ vanishes for a broad range of spectral distribution having divergent mean-square gradients, $\left\langle|\nabla \theta|^{2}\right\rangle \rightarrow \infty$. This includes all 
$\Theta(k)$ no shallower than $k^{-1}$ for large $k$. This type of distribution may be called "regular". The type of "irregular" distribution that gives rise to a non-zero ratio $\left\langle|\nabla \theta|^{2}\right\rangle^{2} /\left\langle|\Delta \theta|^{2}\right\rangle$ in the limit $\left\langle|\nabla \theta|^{2}\right\rangle \rightarrow \infty$ includes power-law spectra shallower than $k^{-1}$ and non-power-law spectra having a significant fraction of $\left\langle\theta^{2}\right\rangle$ at small scales. This fraction must be greater than that allowed by a $k^{-1}$ spectrum and nondiminishing. A more quantitative sense of this fraction is given in what follows. For a finite (but otherwise arbitrarily large) wave number $k_{*}$, let $\theta=\theta_{<}+\theta_{>}$, where the large-scale component $\theta_{<}$and small-scale component $\theta_{>}$are defined by

$$
\theta_{<}=\sum_{k<k_{*}} \widehat{\theta}(\boldsymbol{k}) \exp \{i \boldsymbol{k} \cdot \boldsymbol{x}\}
$$

and

$$
\theta_{>}=\sum_{k \geq k_{*}} \widehat{\theta}(\boldsymbol{k}) \exp \{i \boldsymbol{k} \cdot \boldsymbol{x}\},
$$

respectively. In terms of these components, the ratio $\left\langle|\nabla \theta|^{2}\right\rangle^{2} /\left\langle|\Delta \theta|^{2}\right\rangle$ is given by

$$
\begin{aligned}
\frac{\left\langle|\nabla \theta|^{2}\right\rangle^{2}}{\left\langle|\Delta \theta|^{2}\right\rangle} & =\frac{\left(\left\langle\left|\nabla \theta_{<}\right|^{2}\right\rangle+\left\langle\left|\nabla \theta_{>}\right|^{2}\right\rangle\right)^{2}}{\left\langle\left|\Delta \theta_{<}\right|^{2}\right\rangle+\left\langle\left|\Delta \theta_{>}\right|^{2}\right\rangle} \\
& =\frac{\left(\frac{\left\langle\left|\nabla \theta_{<}\right|^{2}\right\rangle}{\left\langle\left|\Delta \theta_{>}\right|^{2}\right\rangle^{1 / 2}}+\frac{\left\langle\left|\nabla \theta_{>}\right|^{2}\right\rangle}{\left\langle\left|\Delta \theta_{>}\right|^{2}\right\rangle^{1 / 2}}\right)^{2}}{\frac{\left\langle\left|\Delta \theta_{<}\right|^{2}\right\rangle}{\left\langle\left|\Delta \theta_{>}\right|^{2}\right\rangle}+1} .
\end{aligned}
$$

Now, since $\left\langle\left|\nabla \theta_{<}\right|^{2}\right\rangle \leq k_{*}^{2}\left\langle\theta_{<}^{2}\right\rangle\left\langle\infty\right.$, the limit $\left\langle|\nabla \theta|^{2}\right\rangle \rightarrow \infty$ requires $\left\langle\left|\nabla \theta_{>}\right|^{2}\right\rangle \rightarrow \infty$, which in turn entails $\left\langle\left|\Delta \theta_{>}\right|^{2}\right\rangle \rightarrow \infty$ because $\left\langle\left|\Delta \theta_{>}\right|^{2}\right\rangle \geq k_{*}^{2}\left\langle\left|\nabla \theta_{>}\right|^{2}\right\rangle$. Furthermore, $\left\langle\left|\Delta \theta_{<}\right|^{2}\right\rangle \leq k_{*}^{4}\left\langle\theta_{<}^{2}\right\rangle<\infty$. It follows that both $\left\langle\left|\nabla \theta_{<}\right|^{2}\right\rangle /\left\langle\left|\Delta \theta_{>}\right|^{2}\right\rangle^{1 / 2} \rightarrow 0$ and $\left\langle\left|\Delta \theta_{<}\right|^{2}\right\rangle /\left\langle\left|\Delta \theta_{>}\right|^{2}\right\rangle \rightarrow 0$. Hence, if the ratio $\left\langle|\nabla \theta|^{2}\right\rangle^{2} /\left\langle|\Delta \theta|^{2}\right\rangle$ remains non-zero as $\left\langle|\nabla \theta|^{2}\right\rangle \rightarrow \infty$, say bounded away from zero by $\epsilon>0$, then from (11), it is readily deduced that

$$
\epsilon \leq \frac{\left\langle|\nabla \theta|^{2}\right\rangle^{2}}{\left\langle|\Delta \theta|^{2}\right\rangle} \longrightarrow \frac{\left\langle\left|\nabla \theta_{>}\right|^{2}\right\rangle^{2}}{\left\langle\left|\Delta \theta_{>}\right|^{2}\right\rangle}
$$

It follows that

$$
\left\langle\theta_{>}^{2}\right\rangle \geq \frac{\left\langle\left|\nabla \theta_{>}\right|^{2}\right\rangle^{2}}{\left\langle\left|\Delta \theta_{>}\right|^{2}\right\rangle} \geq \epsilon,
$$

where the left-hand side inequality is the familiar Cauchy-schwarz inequality for the small-scale component $\theta_{>}$. Eq. (13) gives an explicit bound for $\left\langle\theta_{>}^{2}\right\rangle$ in terms of $\epsilon$. Thus, the variance at small scales $k \geq k_{*}$ is bounded away from zero if the ratio $\left\langle|\nabla \theta|^{2}\right\rangle^{2} /\left\langle|\Delta \theta|^{2}\right\rangle$ is to remain non-zero in the limit $\left\langle|\nabla \theta|^{2}\right\rangle \rightarrow \infty$.

\section{Main results}

This section derives the main results of this study, including the proof of absence of diffusion anomaly for a broad range of scalar distribution mentioned earlier in $\S 1$. The results are then discussed in the light of existing literature, suggesting exponential decay behavior $\left\langle\theta^{2}\right\rangle \sim e^{-\gamma t}$ in the large-time regime, where $\gamma>0$ is independent of $\kappa$. Finally, the analysis is extended to include a scalar source. 
For this case, the scalar variance is found to become unbounded in forced-dissipative equilibrium in the asymptotic limit.

The notion of diffusion anomaly involves subtle issues, which might be worthy of some clarification. As the product $\kappa\left\langle|\nabla \theta|^{2}\right\rangle$ would trivially vanish in the limit $\kappa \rightarrow 0$ if $\left\langle|\nabla \theta|^{2}\right\rangle\langle\infty$, both the limits $\kappa \rightarrow 0$ and $\left\langle|\nabla \theta|^{2}\right\rangle \rightarrow \infty$ are key features. Furthermore, since $\left\langle|\nabla \theta|^{2}\right\rangle$ may not diverge in finite times, the limit $t \rightarrow \infty$ may also be involved. As it turns out in the present study, $\left\langle|\nabla \theta|^{2}\right\rangle$ does not blow up in finite times. Hence, the limit $t \rightarrow \infty$ is indeed another key element of the problem. Now, the question is in what manner the limit $\kappa \rightarrow 0$ should be considered to account for the associated divergence of $\left\langle|\nabla \theta|^{2}\right\rangle$ in infinite time.

This study takes an appropriate approach that answers the above question satisfactorily. Given fixed $\kappa>0$, let us consider the global maximum of the mean-square scalar gradients $\left\langle\left|\nabla \theta_{T}\right|^{2}\right\rangle$, where $\theta_{T}=\theta(\boldsymbol{x}, T)$ and $T$ denotes the corresponding time upon which this maximum is achieved. In general, $T$ depends on $\kappa$ and is expected to diverge in the limit $\kappa \rightarrow 0$ as discussed in the preceding paragraph. The present study does not attempt to determine how $T$ depends on $\kappa$, except that the hypothetical scaling $T \approx \ln P_{r}$ is examined later on in $\S 3.4$, where a related issue is discussed. The behavior of $\kappa\left\langle\left|\nabla \theta_{T}\right|^{2}\right\rangle$ is then monitored against all admissible distribution $\theta_{T}$ and its upper bounds are deduced in a $\kappa$-independent manner. Being derived in this way, these bounds are valid for all $\kappa$, including the limit $\kappa \rightarrow 0$. Evidently, both limits $t \rightarrow \infty$ and $\left\langle|\nabla \theta|^{2}\right\rangle \rightarrow \infty$ are being nested within the limit $\kappa \rightarrow 0$. Hence, the subsequent constraints on diffusion anomaly deduced from these bounds appropriately take into account the divergence of $\left\langle|\nabla \theta|^{2}\right\rangle$ in infinite time. In essence, this approach is consistent with the double limit $\lim _{\kappa \rightarrow 0} \lim _{t \rightarrow \infty}$ taken in a selective way, in the sense that a dynamical property (here being the peak mean-square gradients) is registered during the first limit $t \rightarrow \infty$ and then monitored during the second limit $\kappa \rightarrow 0$. Evidently, without such a selection, this double limit would yield a trivial result because no smooth scalar distribution would survive the limit $t \rightarrow \infty$ before $\kappa$ tends to zero. In passing, it is worth mentioning that the asymptotic regime of small but positive $\kappa$ and large but finite time, presumably $t \gg T$, is relevant in the study of exponential decay behavior mentioned in the opening paragraph of this section.

\subsection{Production and dissipation of scalar gradients}

The evolution of $\nabla \theta$ is governed by

$$
\partial_{t} \nabla \theta+(\boldsymbol{u} \cdot \nabla) \nabla \theta=\boldsymbol{\omega} \times \nabla \theta-(\nabla \theta \cdot \nabla) \boldsymbol{u}+\kappa \Delta \nabla \theta, \quad \nabla \cdot \boldsymbol{u}=0,
$$

where $\boldsymbol{\omega}=\nabla \times \boldsymbol{u}$ is the vorticity. Apart from the "Coriolis" term $\boldsymbol{\omega} \times \nabla \theta$, whose sole effect is to rotate $\nabla \theta$ without changing its magnitude, Eq. (14) resembles the three-dimensional vorticity equation in several aspects. For example, the gradient $\nabla \theta$ is advected by the velocity field $\boldsymbol{u}$ just as the vorticity $\boldsymbol{\omega}$. As another example, the term $(\nabla \theta \cdot \nabla) \boldsymbol{u}$, which is responsible for the production of scalar gradients, is an analogue of the vortex stretching term. Despite these apparent similarities, there are fundamental differences between "genuine" turbulence and passive scalar advection. First, the passive vector $\nabla \theta$ is irrotational whereas $\boldsymbol{\omega}$ is solenoidal. Second, $\theta$ is materially conserved while there exists no such analogue in the vorticity dynamics. Third, genuine turbulence presumably becomes "more turbulent" in the inviscid limit while the limit of zero diffusivity in scalar advection does not make the advecting flows more effective stirrers. Among these differences, the third one appears to be most prominent, 
directly related to the active (and nonlinear) and passive (and linear) dynamical nature of $\boldsymbol{\omega}$ and $\nabla \theta$, respectively.

Multiplying (14) by $\nabla \theta$ and taking the domain average of the resulting equation yields

$$
\begin{aligned}
\frac{1}{2} \frac{d}{d t}\left\langle|\nabla \theta|^{2}\right\rangle & =-\langle\nabla \theta \cdot(\nabla \theta \cdot \nabla) \boldsymbol{u}\rangle-\kappa\left\langle|\Delta \theta|^{2}\right\rangle \\
& \leq\|\nabla \boldsymbol{u}\|_{\infty}\left\langle|\nabla \theta|^{2}\right\rangle-\kappa\left\langle|\Delta \theta|^{2}\right\rangle
\end{aligned}
$$

where the advection and rotation terms identically vanish and the inequality is straightforward. Here $\|\nabla \boldsymbol{u}\|_{\infty}$ denotes the supremum of $\nabla \boldsymbol{u}$ and, in this study, is assumed to be bounded. This is the maximal degree of smoothness requiring of $\boldsymbol{u}$. Given an initially smooth $\theta$, the manner in which $\left\langle|\nabla \theta|^{2}\right\rangle$ grows as $\kappa$ is decreased determines the outcome of the decay rate $2 \kappa\left\langle|\nabla \theta|^{2}\right\rangle$. This depends primarily on how rapidly $\left\langle|\Delta \theta|^{2}\right\rangle$ grows. Note that even though $\left\langle|\Delta \theta|^{2}\right\rangle$ cannot be controlled a priori, it does good rather than harm, effectively giving rise to the boundedness of $\left\langle|\nabla \theta|^{2}\right\rangle$ for $\kappa>0$. This will become apparent in the next subsection.

A well-known fact which will be exploited later is that for sufficiently small $\kappa,\left\langle|\nabla \theta|^{2}\right\rangle$ grows exponentially in time for an extended period in the initial stage until diffusive effects become significant. For bounded $\|\nabla \boldsymbol{u}\|_{\infty}$, it turns out that more rapid growth is not possible, even in the limit $\kappa \rightarrow 0$. This is apparent from the inherent linearity of (14) and can be stated more quantitatively in the following estimate, being obtained by integrating (15),

$$
\left\langle|\nabla \theta|^{2}\right\rangle \leq\left\langle\left|\nabla \theta_{0}\right|^{2}\right\rangle \exp \left\{2 \int_{0}^{t}\|\nabla \boldsymbol{u}\|_{\infty} d s\right\} \leq\left\langle\left|\nabla \theta_{0}\right|^{2}\right\rangle \exp \{2 \Omega t\}
$$

Here $\theta_{0}=\theta(\boldsymbol{x}, 0)$ and $\Omega$ is an upper bound for $\|\nabla \boldsymbol{u}\|_{\infty}$. Eq. (16) allows $\left\langle|\nabla \theta|^{2}\right\rangle$ to grow exponentially in time at a rate no greater than $2 \Omega$ and forbids any possibility of a finite-time divergence.

\subsection{Bounds for the variance decay rate}

For a preliminary estimate of the decay rate, let us consider (15) in the form

$$
\begin{aligned}
\frac{1}{2} \frac{d}{d t}\left\langle|\nabla \theta|^{2}\right\rangle & \leq\|\nabla \boldsymbol{u}\|_{\infty}\left\langle|\nabla \theta|^{2}\right\rangle-\kappa\left\langle|\Delta \theta|^{2}\right\rangle \\
& =\frac{\left\langle|\Delta \theta|^{2}\right\rangle}{\left\langle|\nabla \theta|^{2}\right\rangle}\left(\Omega \frac{\left\langle|\nabla \theta|^{2}\right\rangle^{2}}{\left\langle|\Delta \theta|^{2}\right\rangle}-\kappa\left\langle|\nabla \theta|^{2}\right\rangle\right) \\
& \leq \frac{\left\langle|\Delta \theta|^{2}\right\rangle}{\left\langle|\nabla \theta|^{2}\right\rangle}\left(\Omega\left\langle\theta^{2}\right\rangle-\kappa\left\langle|\nabla \theta|^{2}\right\rangle\right)
\end{aligned}
$$

where (7) with $\alpha=0$ has been applied to the second equation. The term $\Omega\left\langle\theta^{2}\right\rangle$ is bounded and decreases monotonically. The relevant initial data is such that $\left\langle|\nabla \theta|^{2}\right\rangle$ grows initially. This condition will be assumed throughout this section. Recall that $t=T$ denotes the time when $\left\langle|\nabla \theta|^{2}\right\rangle$ achieves its global maximum. This means that at $t=T, d\left\langle|\nabla \theta|^{2}\right\rangle / d t=0$. Eq. (17) then implies that

$$
\kappa\left\langle\left|\nabla \theta_{T}\right|^{2}\right\rangle \leq \Omega\left\langle\theta_{T}^{2}\right\rangle
$$

This provides an upper bound for (half) the peak decay rate. Now, since $\left\langle|\nabla \theta|^{2}\right\rangle \leq\left\langle\left|\nabla \theta_{T}\right|^{2}\right\rangle$ for all $t \geq 0$ and $\left\langle\theta_{T}^{2}\right\rangle \leq\left\langle\theta_{0}^{2}\right\rangle$, one can deduce that $\kappa\left\langle|\nabla \theta|^{2}\right\rangle \leq \Omega\left\langle\theta_{T}^{2}\right\rangle \leq \Omega\left\langle\theta_{0}^{2}\right\rangle$ for all $t \geq 0$. 
Subject to certain conditions on the spectral distribution of $\theta_{T}$, which will be stated in due course, refined estimates for $\kappa\left\langle\left|\nabla \theta_{T}\right|^{2}\right\rangle$ can be derived. These conditions are admissible but not necessarily realizable, except possibly for the Batchelor spectrum, which has been supported by ample numerical and theoretical evidence. Hence, the results are conditional and as such should be interpreted accordingly. Applying (7) to the second equation of (17) yields

$$
\begin{aligned}
\frac{1}{2} \frac{d}{d t}\left\langle|\nabla \theta|^{2}\right\rangle & \leq \frac{\left\langle|\Delta \theta|^{2}\right\rangle}{\left\langle|\nabla \theta|^{2}\right\rangle}\left(\Omega \frac{\left\langle\left|(-\Delta)^{\alpha / 2} \theta\right|^{2}\right\rangle^{1 /(1-\alpha)}}{\left\langle|\nabla \theta|^{2}\right\rangle^{\alpha /(1-\alpha)}}-\kappa\left\langle|\nabla \theta|^{2}\right\rangle\right) \\
& =\frac{\left\langle|\Delta \theta|^{2}\right\rangle}{\left\langle|\nabla \theta|^{2}\right\rangle^{1 /(1-\alpha)}}\left(\Omega\left\langle\left|(-\Delta)^{\alpha / 2} \theta\right|^{2}\right\rangle^{1 /(1-\alpha)}-\kappa\left\langle|\nabla \theta|^{2}\right\rangle^{1 /(1-\alpha)}\right) .
\end{aligned}
$$

In (19), the terms $\Omega\left\langle\left|(-\Delta)^{\alpha / 2} \theta\right|^{2}\right\rangle^{1 /(1-\alpha)}$ and $\kappa\left\langle|\nabla \theta|^{2}\right\rangle^{1 /(1-\alpha)}$ effectively represent the relative strength of advective production and diffusive suppression of $\left\langle|\nabla \theta|^{2}\right\rangle$, respectively. Their balance shapes up the picture of variance decay. As will be seen presently, this balance is shifted in favor of the latter for steeper $\Theta(k)$. Applying the arguments in the derivation of (18) to (19) yields

$$
\kappa\left\langle\left|\nabla \theta_{T}\right|^{2}\right\rangle^{1 /(1-\alpha)} \leq \Omega\left\langle\left|(-\Delta)^{\alpha / 2} \theta_{T}\right|^{2}\right\rangle^{1 /(1-\alpha)} .
$$

Now, let us suppose that there exists an $\alpha_{0} \in(0,1)$ independent of $\kappa$ such that

$$
\left\langle\left|(-\Delta)^{\alpha / 2} \theta_{T}\right|^{2}\right\rangle<\infty, \text { for } \alpha \leq \alpha_{0} .
$$

It can be seen that if in the limit $\kappa \rightarrow 0, \Theta_{T}(k)=\Theta(k, T)$ remains steeper than $k^{-1}$ for large $k$, such an $\alpha_{0}$ would exist. Given (21) and upon replacing $\alpha$ by $\alpha_{0}$, Eq. (20) becomes

$$
\kappa\left\langle\left|\nabla \theta_{T}\right|^{2}\right\rangle^{1 /\left(1-\alpha_{0}\right)} \leq \Omega\left\langle\left|(-\Delta)^{\alpha_{0} / 2} \theta_{T}\right|^{2}\right\rangle^{1 /\left(1-\alpha_{0}\right)},
$$

or equivalently

$$
\kappa\left\langle\left|\nabla \theta_{T}\right|^{2}\right\rangle \leq \Omega^{\left(1-\alpha_{0}\right)}\left\langle\left|(-\Delta)^{\alpha_{0} / 2} \theta_{T}\right|^{2}\right\rangle \kappa^{\alpha_{0}}
$$

This gives an upper bound for $\kappa\left\langle\left|\nabla \theta_{T}\right|^{2}\right\rangle$ in terms of $\kappa$, among other things, particularly $\alpha_{0}$. The divergence of $\left\langle\left|\nabla \theta_{T}\right|^{2}\right\rangle$ obeys the constraint

$$
\left\langle\left|\nabla \theta_{T}\right|^{2}\right\rangle \leq \Omega^{\left(1-\alpha_{0}\right)}\left\langle\left|(-\Delta)^{\alpha_{0} / 2} \theta_{T}\right|^{2}\right\rangle \kappa^{\left(\alpha_{0}-1\right)} .
$$

A remarkable but not surprising feature of (23) is the explicit dependence on $\alpha_{0}$, which could be termed the degree of smoothness of $\theta_{T}$. As it stands, Eq. (23) reflects the obvious physical connection between smoothness and diffusion that smoother scalar fields diffuse more slowly. Note that (23) also applies to the border case $\alpha_{0}=1$. This case is trivial, simply implying that $\kappa\left\langle\left|\nabla \theta_{T}\right|^{2}\right\rangle$ scales as $\kappa$ if $\left\langle\left|\nabla \theta_{T}\right|^{2}\right\rangle<\infty$. Note also that the conditional bound (23) is an improvement over the result of Tran and Dritschel ${ }^{23}$ for passive scalar in two dimensions and active potential vorticity in various geophysical fluid models. A similar improvement is also possible for their case of enstrophy dissipation in two-dimensional Navier-Stokes turbulence, for which the assumption $\|\nabla \boldsymbol{u}\|_{\infty}<\infty$ (which is not justifiable) is replaced at a minimal cost by the natural condition of finite vorticity (which is materially conserved). By replacing $\theta$ in (7) and (21) by the vorticity and applying the resulting equations to their Eq. (3.2), one can deduce an upper bound for the maximum enstrophy dissipation rate that 
scales as $\nu^{\alpha_{0} /\left(2-\alpha_{0}\right)}$. This scaling converges to zero more slowly than the scaling $\kappa^{\alpha_{0}}$. The slower convergence is the cost for relaxing the condition $\|\nabla \boldsymbol{u}\|_{\infty}<\infty$.

On physical grounds, $\Theta_{T}(k)$ can become shallower as $\kappa$ is decreased. This means that condition (21) may not be fulfilled, i.e. there may exist no $\alpha>0$ independent of $\kappa$ such that $\left\langle\left|(-\Delta)^{\alpha} \theta_{T}\right|^{2}\right\rangle<\infty$. If this is the case, the scalar field may be said to become virtually singular. This includes the Batchelor spectrum $\Theta(k) \propto k^{-1}$, which has been believed to be the correct scaling of the so-called viscousconvective range, i.e. the range between the energy dissipation scale and the scalar diffusion scale. A plausible scenario is that $\Theta_{T}(k)$ becomes increasingly shallower, approaching the limiting scaling $k^{-1}$ in the limit $\kappa \rightarrow 0$. For this case, applying (8) to the second equation of (17) yields

$$
\begin{aligned}
\frac{1}{2} \frac{d}{d t}\left\langle|\nabla \theta|^{2}\right\rangle & \leq \frac{\left\langle|\Delta \theta|^{2}\right\rangle}{\left\langle|\nabla \theta|^{2}\right\rangle}\left(\frac{\Omega\left\langle\theta^{2}\right\rangle}{\ln \left(k_{1} / k_{2}\right)}-\kappa\left\langle|\nabla \theta|^{2}\right\rangle\right) \\
& \leq \frac{\left\langle|\Delta \theta|^{2}\right\rangle}{\left\langle|\nabla \theta|^{2}\right\rangle}\left(\frac{\Omega\left\langle\theta^{2}\right\rangle}{\ln \left(k_{\kappa} / k_{\nu}\right)}-\kappa\left\langle|\nabla \theta|^{2}\right\rangle\right),
\end{aligned}
$$

where $k_{1}$ and $k_{2}$ have been replaced by the viscous dissipation wave number $k_{\nu}$ and the diffusion wave number $k_{\kappa}$, respectively. Following Tran and Dritschel, ${ }^{23}$ let us identify the ratio $k_{\kappa}^{2} / k_{\nu}^{2}$ with the Prandtl or Schmidt number $P_{r}$. By substituting $P_{r}=k_{\kappa}^{2} / k_{\nu}^{2}$ into (25), one obtains

$$
\frac{1}{2} \frac{d}{d t}\left\langle|\nabla \theta|^{2}\right\rangle \leq \frac{\left\langle|\Delta \theta|^{2}\right\rangle}{\left\langle|\nabla \theta|^{2}\right\rangle}\left(\frac{2 \Omega\left\langle\theta^{2}\right\rangle}{\ln P_{r}}-\kappa\left\langle|\nabla \theta|^{2}\right\rangle\right) .
$$

It follows that

$$
\kappa\left\langle\left|\nabla \theta_{T}\right|^{2}\right\rangle \leq \frac{2 \Omega\left\langle\theta_{T}^{2}\right\rangle}{\ln P_{r}}
$$

Eq. (27) implies an extraordinarily weak dependence of $\kappa\left\langle\left|\nabla \theta_{T}\right|^{2}\right\rangle$ on $P_{r}$. Nevertheless, $\kappa\left\langle\left|\nabla \theta_{T}\right|^{2}\right\rangle$ vanishes in the limit $P_{r} \rightarrow \infty$. Similar lines of calculation ${ }^{23}$ for the case of enstrophy decay in twodimensional Navier-Stokes turbulence have found that the enstrophy dissipation rate scales with the Reynolds number $R e$ as $(\ln R e)^{-1 / 2}$. Again, this relatively slower convergence to zero (as compared with $\left.\left(\ln P_{r}\right)^{-1}\right)$ is the cost for relaxing the condition $\|\nabla \boldsymbol{u}\|_{\infty}<\infty$.

\subsection{Discussion}

The results in the preceding subsection cover a broad class of scalar distribution corresponding to no anomalous diffusion. This class of regular distribution includes power-law spectra no shallower than $k^{-1}$. The type of "singular" distribution that would give rise to diffusion anomaly would feature a significant fraction of $\left\langle\theta^{2}\right\rangle$ "cascading" to the vicinity of an ever-increasing diffusion wave number $k_{\kappa} \propto \kappa^{-1 / 2}$ as $\kappa \rightarrow 0$. The decay rate would then be proportional to this fraction. As can be seen from the estimates of $\S 2$, this fraction would have to be greater than that allowed by the Batchelor $k^{-1}$ spectrum. This is consistent with recent results ${ }^{1,7,14,19}$ suggesting spectra shallower than $k^{-1}$. Note that for such spectra, virtually all (not just a fraction) of $\left\langle\theta^{2}\right\rangle$ is at the small scales.

A number of studies ${ }^{8,13,17,20}$ have suggested that in the large-time regime, the variance decays as $\left\langle\theta^{2}\right\rangle \propto e^{-\gamma t}$, where $\gamma>0$ is independent of $\kappa$. The exponential decay rate $\gamma$ differs from the usual decay rate $2 \kappa\left\langle|\nabla \theta|^{2}\right\rangle$ and is given by

$$
\gamma=-\frac{1}{\left\langle\theta^{2}\right\rangle} \frac{d}{d t}\left\langle\theta^{2}\right\rangle=\frac{2 \kappa\left\langle|\nabla \theta|^{2}\right\rangle}{\left\langle\theta^{2}\right\rangle} .
$$


In the presence of diffusion anomaly (the exceptional case of singular distribution mentioned above), this large-time regime presumably corresponds to $t \approx T\left(\kappa_{0}\right)$ for some small $\kappa=\kappa_{0}$, beyond which $\kappa\left\langle\left|\nabla \theta_{T}\right|^{2}\right\rangle$ becomes independent of $\kappa$. If $\gamma$ stays approximately constant for a period of time, then the ratio $\left\langle|\nabla \theta|^{2}\right\rangle /\left\langle\theta^{2}\right\rangle$ is required to remain approximately unchanged, i.e. both $\left\langle|\nabla \theta|^{2}\right\rangle$ and $\left\langle\theta^{2}\right\rangle$ decay approximately at the same rate $\gamma$. In the absence of diffusion anomaly, $\gamma$ vanishes in the same manner as the usual decay rate for $t \approx T$. For example, for the Batchelor spectrum, Eqs. (27) and (28) imply that the exponential decay rate at $t=T$, denoted by $\gamma_{T}$, satisfies

$$
\gamma_{T} \leq \frac{4 \Omega}{\ln P_{r}}
$$

Although $\gamma_{T}$ depends on $P_{r}$ and vanishes in the limit $P_{r} \rightarrow \infty$, in principle $\gamma$ might become sizable for $t \gg T$, provided that the ratio $\left\langle|\nabla \theta|^{2}\right\rangle /\left\langle\theta^{2}\right\rangle$ has increased by a $\ln P_{r}$-fold since $t=T$. This requires of the variance itself a decrease of at least a $\ln P_{r}$-fold, i.e. $\left\langle\theta^{2}\right\rangle \leq\left\langle\theta_{T}^{2}\right\rangle / \ln P_{r}$, because $\left\langle|\nabla \theta|^{2}\right\rangle$ also decays (at least on average if not monotonically). Note that an increase of $\left\langle|\nabla \theta|^{2}\right\rangle /\left\langle\theta^{2}\right\rangle$ requires that $\left\langle\theta^{2}\right\rangle$ decay relatively more rapidly than $\left\langle|\nabla \theta|^{2}\right\rangle$. This means that the advective production of scalar gradients remains considerably active throughout the regime $t>T$.

An interesting problem arises from this analysis is that regardless of the realizability of diffusion anomaly, the cumulative decay, up to $t=T$, defined by

$$
\left\langle\theta_{0}^{2}\right\rangle-\left\langle\theta_{T}^{2}\right\rangle=2 \kappa \int_{0}^{T}\left\langle|\nabla \theta|^{2}\right\rangle d t
$$

may remain non-zero and be independent of $\kappa$. A similar problem on the cumulative decay of enstrophy in freely evolving two-dimensional turbulence has been briefly addressed in a recent numerical study, ${ }^{26}$ illustrating the absence of enstrophy dissipation anomaly. There, it was observed that by the time $T(R e)$ of peak enstrophy dissipation, the enstrophy loss was significant and, more importantly, roughly the same for a range of Reynolds numbers $R e$. It was also noted that $T(R e)$ increased with $R e$, probably as $\ln R e$. In the light of these results, it seems plausible that the cumulative decay, defined by (30), remains non-zero in the limit $\kappa \rightarrow 0$. For a quantitative sense of how this might be the case, let us consider the following picture, which is in accord with the above observations for the decay of two-dimensional enstrophy. In accord with (16), $\left\langle|\nabla \theta|^{2}\right\rangle$ is expected to grow exponentially for a good part of the time interval $[0, T]$, before diffusive suppression becomes significant toward the end. Hence, one may write $\left\langle|\nabla \theta|^{2}\right\rangle \approx\left\langle\left|\nabla \theta_{0}\right|^{2}\right\rangle \exp \left\{c_{1} \Omega t\right\}$, where $c_{1}>0$ is a dimensionless constant. Given this growth, the requirement of $T$ for the right-hand side of (30) to be independent of $\kappa$ is $T \approx c_{1}^{-1} \Omega^{-1} \ln \left(c_{2} P_{r}\right)$, where $c_{2}>0$ is another dimensionless constant. Indeed, by substituting these into (30), the integral on the right-hand side can be readily evaluated, and the result is $\propto P_{r}$. This effectively cancels out the diffusion coefficient in (30). Thus the cumulative decay could be non-zero and independent of $\kappa$ in the limit $\kappa \rightarrow 0$. In the absence of diffusion anomaly, one may expect this picture to be most plausible for the Batchelor spectrum. The reason is that diffusive suppression of scalar gradients becomes relatively stronger (as compared with their advective production) for steeper spectra, as suggested by the analysis in $\S 3.2$. This means that for power-law spectra no shallower than $k^{-1}$, the $k^{-1}$ spectrum is most favorable for the gradient production and therefore highly likely to return the most cumulative decay. 


\subsection{The forced case}

This subsection considers the dynamical picture of forced-dissipative equilibrium in the limit $\kappa \rightarrow 0$, where the scalar variance is perpetually replenished at the large scales by a scalar source. In the presence of such a source, the absence of diffusion anomaly would be catastrophic, in the sense that the scalar field would diverge, particularly at the injection scales. It turns out that for power-law spectra, $\left\langle\theta^{2}\right\rangle$ diverges, regardless of whether or not diffusion anomaly is realizable. The classical

spectrum $\Theta(k) \propto k^{-1}$ is the steepest one that could remain bounded while still satisfying the condition $\left\langle\theta^{2}\right\rangle \rightarrow \infty$. Steeper spectra would necessarily grow without bound, i.e. blowup at large scales.

With the addition of a scalar source $f$ to the right-hand side of the advection-diffusion equation in (1), Eqs. (2) and (15) become

$$
\frac{1}{2} \frac{d}{d t}\left\langle\theta^{2}\right\rangle=-\kappa\left\langle|\nabla \theta|^{2}\right\rangle+\langle\theta f\rangle
$$

and

$$
\frac{1}{2} \frac{d}{d t}\left\langle|\nabla \theta|^{2}\right\rangle \leq \frac{\left\langle|\Delta \theta|^{2}\right\rangle}{\left\langle|\nabla \theta|^{2}\right\rangle}\left(\Omega \frac{\left\langle|\nabla \theta|^{2}\right\rangle^{2}}{\left\langle|\Delta \theta|^{2}\right\rangle}+\frac{\langle\nabla \theta \cdot \nabla f\rangle\left\langle|\nabla \theta|^{2}\right\rangle}{\left\langle|\Delta \theta|^{2}\right\rangle}-\kappa\left\langle|\nabla \theta|^{2}\right\rangle\right),
$$

respectively. Let us consider a persistent source as described above, i.e. $\langle\theta f\rangle>0$, and forceddissipative equilibrium dynamics, i.e. $\kappa\left\langle|\nabla \theta|^{2}\right\rangle=\langle\theta f\rangle$. These can be understood in an average sense and are assumed for all $P_{r}$. Suppose that in the limit $\kappa \rightarrow 0,\left\langle\theta^{2}\right\rangle$ remains bounded. Then for powerlaw scaling, $\Theta(k)$ is steeper than $k^{-1}$. From the above calculation for the unforced case, the first term in the brackets on the right-hand side of (32) vanishes as $\left\langle|\nabla \theta|^{2}\right\rangle \rightarrow \infty$. The second term also vanishes for a broad class of scalar sources $f$, particularly those satisfying $\left\langle|\nabla f|^{2}\right\rangle<\infty$. It follows that for $\langle\theta f\rangle>0$, the assumed forced-dissipative equilibrium $\kappa\left\langle|\nabla \theta|^{2}\right\rangle=\langle\theta f\rangle$ cannot be achieved. This means that such an equilibrium requires $\left\langle\theta^{2}\right\rangle \rightarrow \infty$. The spectrum $\Theta(k)=c k^{-1}$, extending to infinity and having non-diminishing $c$, can be seen as a possible solution, corresponding to a logarithmic divergence of the scalar variance toward the small scales. This singular distribution is special and anomalously diffused. Other cases of blowup via unbounded spectra steeper than $k^{-1}$ are also permitted. These may or may not be accompanied by diffusion anomaly. Note that non-power-law spectra having significant variance at ever-smaller scales and $\left\langle\theta^{2}\right\rangle<\infty$ cannot be ruled out. Similar arguments have been used by $\operatorname{Tran}^{27}$ for the problem of enstrophy dissipation in forced-dissipative two-dimensional turbulence (in an essentially unbounded domain). There, the vorticity was found to diverge in the inviscid limit.

\section{Conclusion}

In conclusion, this study has derived upper bounds for the peak decay rate of the variance of a passive scalar $\theta$ advected by three-dimensional flows having bounded velocity gradients $|\nabla \boldsymbol{u}|<\infty$. These bounds are expressible in terms of controlled physical parameters and conditional on the distribution of the scalar field $\theta_{T}=\theta(\boldsymbol{x}, T)$ at the time of peak decay $t=T$. It has been found that in the limit of vanishingly small diffusivity $\kappa$, the peak decay rate vanishes as $\kappa^{\alpha_{0}}$ if there exists an $\alpha_{0} \in(0,1]$ independent of $\kappa$ such that $\left\langle\left|(-\Delta)^{\alpha / 2} \theta_{T}\right|^{2}\right\rangle<\infty$ for $\alpha \leq \alpha_{0}$. This condition is satisfied if at $t=T$, the variance spectrum $\Theta_{T}(k)$ is steeper than $k^{-1}$ at the spectral tail, i.e. at large wave numbers $k$. Such a positive $\alpha_{0}$ is not known to exist as numerical simulations ${ }^{28}$ indicate an increasing roughness of the 
scalar field with increasing $P_{r}$. If this hypothesis turns out to be false, a plausible scenario consistent with Batchelor's theory is that $\Theta(k)$ becomes increasingly shallower for smaller $\kappa$, approaching the Batchelor scaling $k^{-1}$ in the limit $\kappa \rightarrow 0$. For this classical spectrum, the peak decay rate also vanishes, albeit more slowly — like $\left(\ln P_{r}\right)^{-1}$. These results rule out anomalous diffusion for a broad range of $\Theta_{T}(k)$, including power-law spectra no shallower than $k^{-1}$. The implication is that a non-vanishing decay rate in the limit $\kappa \rightarrow 0$ necessarily requires the variance at small scales to be greater than that allowed by the Batchelor spectrum. The absence of anomalous diffusion in the above cases can be seen as a consequence of strong diffusive suppression of scalar gradients, which are relatively weakly produced by advection. In the presence of a scalar source, $\left\langle\theta^{2}\right\rangle$ has been found to diverge in the limit $\kappa \rightarrow 0$ if $\Theta(k)$ obeys power-law scalings. Hence, spectra steeper than $k^{-1}$ would necessarily grow without bound. The classical $k^{-1}$ spectrum, being bounded and accompanied by diffusion anomaly, is a possible solution, corresponding to a logarithmic divergence of the variance toward the small scales. Interestingly, a diverging scalar field would be necessary for transferring the injected scalar variance to the small scales for disposal.

The present results have been derived on the assumption of bounded velocity gradients. This assumption is plausible for the Batchelor turbulence regime, where the Prandtl number $P_{r}$ tends to infinity while the Reynolds number $R e$ remains bounded. In fact, there exists neither theoretical nor numerical evidence suggesting otherwise. The case where $R e \rightarrow \infty$ and $P_{r}>0$ is interesting and presumably much more involved. In this case, one would expect the advecting flows to become increasingly "rougher", possibly leading to unbounded velocity gradients in the limit $R e \rightarrow \infty$. Intuitively, diffusion anomaly is more plausible for this case because the scalar gradient production term can no longer be controlled a priori. This is no surprise as the original notion of energy dissipation anomaly is associated with the limit of infinite Reynolds number.

${ }^{1}$ T. M. Antonsen, Jr. Zhencan Fan, E. Ott, and E. Garcia-Lopez, "The role of chaotic orbits in the determination of power spectra of passive scalars," Phys. Fluids 8, 3094 (1996).

${ }^{2}$ E. Balkovsky and A. Fouxon, "Universal long-time properties of Lagrangian statistics in the Batchelor regime and their application to the passive scalar problem," Phys. Rev. E 60, 4164 (1999).

${ }^{3}$ G. K. Batchelor, "Small-scale variation of convected quantities like temperature in turbulent fluid. Part 1. General discussion and the case of small conductivity," J. Fluid Mech. 5, 113 (1959).

${ }^{4}$ G. K. Batchelor I. A. Howell, and A. A. Townsend, "Small-scale variation of convected quantities like temperature in turbulent fluid. Part 2. The case of large conductivity," J. Fluid Mech. 5, 134 (1959). ${ }^{5}$ M. Chertkov, G. Falkovich, and I. Kolokolov, "Intermittent dissipation of a passive scalar in turbulence," Phys. Rev. Lett. 80, 2121 (1998).

${ }^{6} \mathrm{~S}$. Corrsin, "On the spectrum of isotropic temperature fluctuation in isotropic turbulence," J. Appl. Phys. 22, 469 (1951).

${ }^{7}$ D. R. Fereday and P. H. Haynes, "Scalar decay in two-dimensional chaotic advection and Batchelorregime turbulence," Phys. Fluids 16, 4359 (2004).

${ }^{8}$ P. H. Haynes and J. Vanneste, "What controls the decay rate of passive scalar in smooth random flows?" Phys. Fluids 17, 097103 (2005).

${ }^{9}$ R. H. Kraichnan, "Small-scale structure of a scalar field convected by turbulence," Phys. Fluids 11, 945 (1968). 
${ }^{10}$ W. Liu, "Does a fast mixer really exist?" Phys. Rev. E 72, 016312 (2005).

${ }^{11}$ A. D. Majda, "The random uniform shear layer: an explicit example of turbulent diffusion with broad tail probability distributions," Phys. Fluids A 5, 1963 (1993).

${ }^{12}$ A. M. Obukhov, "The structure of the temperature field in a turbulent flow," Izv. Akad. Nauk. SSSR, Ser. Geophys. 13, 58 (1949).

${ }^{13}$ R. T. Pierrehumbert, "Tracer microstructure in the large-eddy dominated regime," Chaos, Solitons Fractals. 4, 1091 (1994).

${ }^{14}$ A. A. Schekochihin, P. H. Haynes, and S. C. Cowley, "Diffusion of passive scalar in a finite random flow," Phys. Rev. E 70, 046304 (2004).

${ }^{15}$ K. R. Sreenivasan, "The passive scalar spectrum and the Obukhov-Corrsin constant," Phys. Fluids 8, 189 (1996).

${ }^{16}$ K. R. Sreenivasan, "On local isotropy of passive scalars in turbulent shear flows," Proc. Roy. Soc. A 434, 165 (1991).

${ }^{17}$ J. Sukhatme and R. T. Pierrehumbert, "Decay of passive scalars under the action of single scale smooth velocity fields in bounded two-dimensional domains: from non-self-similar probability distribution function to self-similar Eigenmodes," Phys. Rev. E 66, 056302 (2002).

${ }^{18}$ C. V. Tran, "An upper bound for passive scalar diffusion in shear flows," Phys. Fluids 19, 068104 (2007).

${ }^{19}$ Y. K. Tsang, T. M. Antonsen, and E. Ott, "Exponential decay of chaotically advected passive scalars in the zero diffusivity limit," Phys. Rev. E 71066301 (2005).

${ }^{20}$ A. Wonhas and J. C. Vassilicos, "Mixing in fully chaotic flows," Phys. Rev. E 66, 051205 (2002).

${ }^{21}$ P. K. Yeung, Shuyi Xu, and K. R. Sreenivasan, "Schmidt number effects on turbulent transport with uniform mean scalar gradient," Phys. Fluids 14, 4178 (2002).

${ }^{22}$ P. A. Davidson, "Turbulence: An Introduction for Scientists and Engineers," Oxford University Press (2004).

${ }^{23}$ C. V. Tran and D. G. Dritschel, "Vanishing enstrophy dissipation in two-dimensional Navier-Stokes turbulence in the inviscid limit," J. Fluid Mech. 559, 107 (2006).

${ }^{24}$ C. V. Tran, "Nonlinear transfer and spectral distribution of energy in $\alpha$ turbulence," Physica D 191, 137 (2004).

${ }^{25}$ C. V. Tran and T. G. Shepherd, "Constraints on the spectral distribution of energy and enstrophy dissipation in forced two-dimensional turbulence," Physica D 165, 199 (2002).

${ }^{26}$ D. G. Dritschel, C. V. Tran and R. K. Scott, "Revisiting Batchelor's theory of two-dimensional turbulence," J. Fluid Mech. 591, 379 (2007).

${ }^{27}$ C. V. Tran, "Constraints on inertial range scaling laws in forced two-dimensional Navier-Stokes turbulence," Phys. Fluids 19, 108109 (2007).

${ }^{28}$ J. Schumacher and K. R. Sreenivasan, "Geometric features of the mixing of passive scalars at high Schmidt numbers," Phys. Rev. Lett. 91, 174501 (2003). 